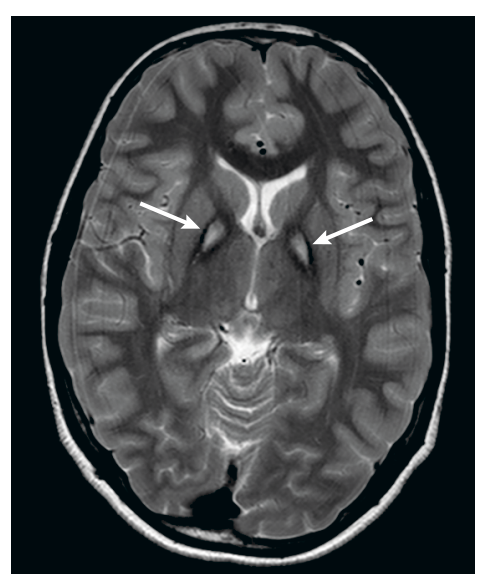

MRI scan showing iron accumulation in the globus pallidus (arrows) in a patient with PKAN. Image courtesy of T. Klopstock, University of Munich, Germany.

earlier intervention could improve the outcome of treatment.

Klopstock points out that there is still more information to be gleaned from these data. "The results of our study, in particular the 18 months

Iron concentrations in the globus pallidus decreased in the group that received deferiprone of treatment in the placebo group, will inform us about the natural history of PKAN, which will shape the design of future trials in this ultra-rare disease."

Sarah Lemprière

ORIGINAL ARTICLE Klopstock, T. et al. Safety and efficacy of deferiprone for pantothenate kinaseassociated neurodegeneration: a randomised, double-blind, controlled trial and an open-label extension study. Lancet Neurol. 18, 631-642 (2019)

In patients with low DAT activity at baseline, information processing speed and patient-reported apathy improved with methylphenidate treatment. By contrast, no changes were seen in these measures in patients with normal DAT activity. The results show that dopamine status can be used to stratify patients for treatment of cognitive impairment after TBI.

In addition, the study design demonstrates how a biomarker that relates to a treatment's mechanism of action can help to identify positive outcomes in trials. "If our trial had been conducted without patient stratification, methylphenidate would have been judged ineffective in the treatment of post-TBI cognitive and behavioural impairments," the researchers say. "This illustrates the importance of considering patient stratification when designing treatment trials in TBI."

lan Fyfe

ORIGINAL ARTICLE Jenkins, P. O. et al. Stratifying drug treatment of cognitive impairments after traumatic brain injury using neuroimaging. Brain https://doi.org/10.1093/ brain/awz149 (2019)
ALZHEIMER DISEASE

\section{Inflammatory pathways relate to early Alzheimer disease pathology}

Key inflammatory pathways that are activated in the mild cognitive impairment (MCl) stage of Alzheimer disease (AD) have been identified in a new study published in Annals of Clinical and Translational Neurology. Inflammatory activation correlated with levels of pathogenic tau and amyloid- $\beta(A \beta)$, providing an opportunity to assess disease severity in the early stages of disease.

A large body of evidence has demonstrated that inflammation has a role in AD. However, details of the inflammatory pathways that are activated in humans are not yet known, and the relationships between peripheral inflammatory changes, CNS inflammation and the $A \beta$ and tau pathology associated with neurodegeneration are not clear.

In the new study, Jagan Pillai and colleagues set out to address these uncertainties by investigating inflammatory markers in patients with $\mathrm{MCl}$. "We detail a framework for investigating the pathophysiology of inflammatory changes in relation to levels of neurodegenerative biomarkers concurrently in the plasma and cerebrospinal fluid," explains Pillai.

The researchers first analysed cerebrospinal fluid (CSF) and plasma from 48 patients with $\mathrm{MCl}$, a diagnosis that had been confirmed by the presence of diagnostic levels of $A \beta_{42}$ and phosphorylated tau in the CSF. "In prior studies of inflammatory changes in $A D$, small numbers of inflammatory analytes were measured, typically ten or fewer," says Pillai. "We used a protein multiplex system to interrogate multiple inflammatory analyte changes of interest."

The analysis showed that levels of several inflammatory proteins in the CSF and plasma correlated with those of total and phosphorylated tau. Similar findings were seen in a validation cohort of 43 participants in the Alzheimer's Disease Neuroimaging Initiative.

Further investigation demonstrated that some groups of analytes correlated with tau and $A \beta_{42}$ more strongly than the constituent analytes alone. Functional pathway analysis of these groups revealed that total and phosphorylated tau levels were associated most strongly with activity of the tumour necrosis factor signalling pathway, and that $A \beta_{42}$ levels were associated most strongly with the complement and coagulation cascade.

As further validation, the team studied the transcriptome of postmortem brain tissue from 19 patients with AD. This analysis revealed enriched expression of genes involved in the same inflammatory pathways.

"Our work shows that analytes from well-described inflammation-related pathways can be identified in tandem in both peripheral and CNS compartments and relate to the severity of neurodegeneration markers," says Pillai.

According to Pillai, further work to understand inflammatory pathway activation at different stages of $A D$ could help to determine when these inflammatory changes are best targeted therapeutically. "In addition, the study leaves open the intriguing connection between the analyte levels and longitudinal changes in cognition, and analysis of this is currently ongoing," Pillai concludes.

Ian Fyfe

ORIGINAL ARTICLE Pillai, J. A. et al. Key inflammatory pathway activations in the MCl stage of Alzheimer's diseaase. Ann. Clin. Transl Neurol. https://doi.org/10.1002/acn3.50827 (2019) 